University of South Florida

DIGITAL COMMONS

Digital Commons @ University of

@ UNIVERSITY OF SOUTH FLORIDA

South Florida

$1-1-2014$

\title{
2014 Work Plan USF Sarasota-Manatee
}

USF

Follow this and additional works at: https://digitalcommons.usf.edu/usf_accountability_reports

\section{Scholar Commons Citation}

USF, "2014 Work Plan USF Sarasota-Manatee" (2014). USF Accountability Reports. 45.

https://digitalcommons.usf.edu/usf_accountability_reports/45

This Article is brought to you for free and open access by the USF Archives at Digital Commons @ University of South Florida. It has been accepted for inclusion in USF Accountability Reports by an authorized administrator of Digital Commons @ University of South Florida. For more information, please contact digitalcommons@usf.edu. 


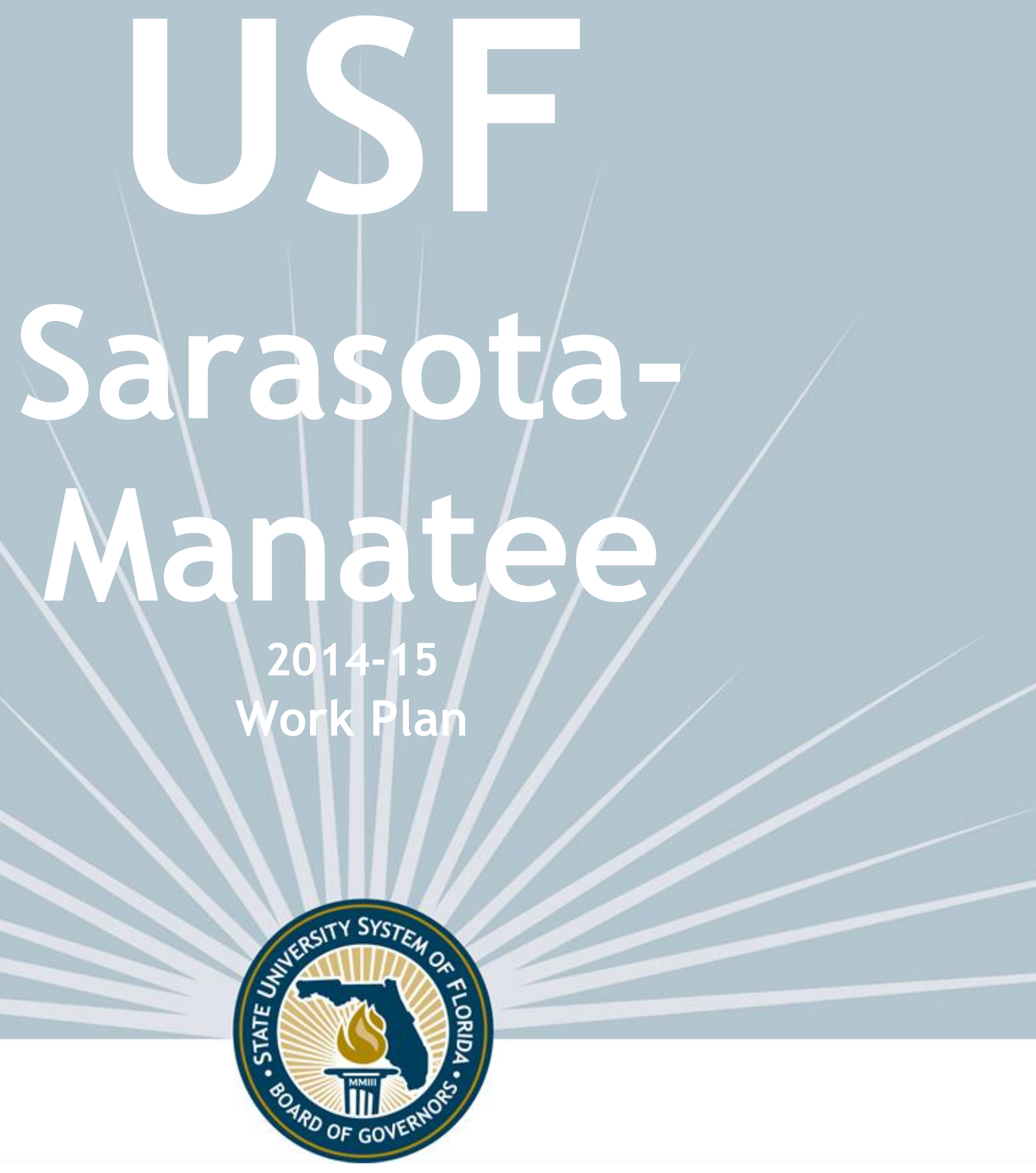

USF Sarasota-Manatee

Work Plan Presentation for 2014-15 Board of Governors Review

STATE UNIVERSITY SYSTEM of FLORIDA Board of Governors 


\section{INTRODUCTION}

The State University System of Florida has developed three tools that aid in guiding the System's future.

1) The Board of Governors' new Strategic Plan 2012-2025 is driven by goals and associated metrics that stake out where the System is headed;

2) The Board's Annual Accountability Report provides yearly tracking for how the System is progressing toward its goals;

3) Institutional Work Plans connect the two and create an opportunity for greater dialogue relative to how each institution contributes to the System's overall vision.

These three documents assist the Board with strategic planning and with setting short-, mid-and long-term goals. They also enhance the System's commitment to accountability and driving improvements in three primary areas of focus: 1) academic quality, 2) operational efficiency, and 3) return on investment.

The Board will use these documents to help advocate for all System institutions and foster even greater coordination with the institutions and their Boards of Trustees.

Once a Work Plan is approved by each institution's respective Boards of Trustees, the Board of Governors will review and consider the plan for potential acceptance of 2014-15 components. Longer-term components will inform future agendas of the Board's Strategic Planning Committee. The Board's acceptance of a work plan does not constitute approval of any particular component, nor does it supersede any necessary approval processes that may be required for each component. 


\section{TABLE OF CONTENTS}

\section{STRATEGY}
a. Mission Statement
b. Vision Statement
c. Statement of Strategy
d. Strengths and Opportunities
e. Key Initiatives \& Investments

\section{PERFORMANCE BASED FUNDING METRICS}

\section{PREEMINENT RESEARCH UNIVERSITY METRICS}

\section{OTHER KEY PERFORMANCE INDICATORS}
a. Goals Common to All Universities
b. Goals Specific to Research Universities
c. Institution Specific Goals

\section{OPERATIONS}
a. Fiscal Information (includes Tuition Differential Fee Request)
b. Enrollment Planning
c. Academic Program Coordination

\section{DEFINITIONS}




\section{MISSION STATEMENT (What is your purpose?)}

The University of South Florida Sarasota-Manatee provides access to professional higher education programs and scholarly research in a personalized living and learning community that graduates successful leaders who empower Florida's Suncoast to thrive locally, nationally, and globally.

\section{VISION STATEMENT (What do you aspire to?)}

The University of South Florida Sarasota-Manatee strives to be a hometown comprehensive institution with ever-increasing impact.

\section{STATEMENT OF STRATEGY (How will you get there?)}

Given your mission, vision, strengths and available resources, provide a brief description of your market and your strategy for addressing and leading it.

The University of South Florida Sarasota-Manatee (USF Sarasota-Manatee) mission is to ensure access to professional higher education, especially to those living in the local Sarasota-Manatee region. The University is pursuing this endeavor through three avenues: 1) partnerships with the State College System; 2) expanding lower-level coursework; and 3) growing its undergraduate and graduate programs to fill local and statewide needs.

The USF Sarasota-Manatee target markets include Florida College System transfers, returning adult students, and local area high school graduates who wish to stay within their community while pursuing a Florida public university education. 


\section{STRENGTHS AND OPPORTUNITIES (within 3 years)}

What are your core capabilities, opportunities and challenges for improvement?

USF Sarasota-Manatee is privileged to provide education that permits students to "learn where they earn," to participate in regional economic development, to link continuing professional training to maintain workforce competence, and to respond to the higher education needs of a growing population. As a member of the USF System, USF Sarasota-Manatee benefits from the efficiency of shared resources, the opportunity for collaboration with other member institutions, and a unified brand that yields identity and impact.

Currently, the University's steepest challenge is providing a campus-life experience for students without residence halls.

\section{KEY INITIATIVES \& INVESTMENTS (within 3 years)}

Describe your top three key initiatives for the next three years that will drive improvement in Academic Quality, Operational Efficiency, and Return on Investment.

1. Continue to improve baccalaureate retention and graduation rates - To improve graduation and retention rates, USFSM has re-organized its Academic and Student Affairs areas to focus on enrollment management, student success, and student engagement. Specific approaches include an "early warning" system for students at academic risk, one-stop information commons for direct student assistance in tutoring, e-learning, writing support and undergraduate research support. Additionally, student engagement, which has been shown to improve graduation and retention rates, has included the adoption of centralized co-curricular activity documentation and communication through the OrgSync campus engagement system. USF Sarasota-Manatee leverages technology to improve studentlearning outcomes and uses tuition differential funds to open additional undergraduate course sections, which can improve rates of graduation.

2. Expand offerings in the STEM fields - Looking to expand offerings in STEM-related fields, USF Sarasota-Manatee will begin a bachelor's of science degree in biology in Fall Semester 2014. Students will take course work for this degree program partially on the campus of Mote Marine Laboratory, a university-community partnership.

3. Identify student residence housing opportunities. Efforts to identify student housing at USFSM are tied to student success and engagement. We have had an increased demand from parents of students who are planning on attending USF Sarasota-Manatee. These families want a real campus-based experience for their children, as research demonstrates those students who are in student are far more likely to complete their degree faster and experience greater student success due in part to the increased access to university systems of support. Therefore, USF Sarasota-Manatee will continue work to identify possible student housing options on or near campus. 
Each university is required to complete the table below, providing their goals for the metrics used in the Performance Based Funding model that the Board of Governors approved at its January 2014 meeting. The Board of Governors will consider the shaded 2014-15 goals for approval.

\begin{tabular}{|c|c|c|c|c|c|c|}
\hline & $\begin{array}{l}\text { ONE-YEAR } \\
\text { TREND }\end{array}$ & $\begin{array}{l}2012-13 \\
\text { ACTUAL }\end{array}$ & $\begin{array}{c}2013-14 \\
\text { ESTIMATES }\end{array}$ & $\begin{array}{l}2014-15 \\
\text { GOALS }\end{array}$ & $\begin{array}{l}2015-16 \\
\text { GOALS }\end{array}$ & $\begin{array}{l}2016-17 \\
\text { GOALS }\end{array}$ \\
\hline \multicolumn{7}{|l|}{ Metrics Common To All Universities } \\
\hline $\begin{array}{l}\text { Percent of Bachelor's Graduates } \\
\text { Employed Full-time in Florida or } \\
\text { Continuing their Education in the U.S. } \\
\text { One Year After Graduation }\end{array}$ & $1 \%$ & $72 \%$ & $74 \%$ & $75 \%$ & $76 \%$ & $76 \%$ \\
\hline $\begin{array}{l}\text { Median Wages of Bachelor's Graduates } \\
\text { Employed Full-time in Florida } \\
\text { One-Year After Graduation }\end{array}$ & $0 \%$ & $\$ 33,300$ & $\$ 33,996$ & $\$ 34,676$ & $\$ 35,370$ & $\$ 36,077$ \\
\hline $\begin{array}{l}\text { Average Cost per Bachelor's Degree } \\
\text { [Instructional Costs to the University] }\end{array}$ & \multicolumn{6}{|c|}{ Reported at the USF System level } \\
\hline $\begin{array}{l}\text { FTIC } 6 \text { year Graduation Rate } \\
\text { [Includes full- and part-time students] }\end{array}$ & $\mathrm{n} / \mathrm{a}$ & $\mathrm{n} / \mathrm{a}$ & $\mathrm{n} / \mathrm{a}$ & $\mathrm{n} / \mathrm{a}$ & $\mathrm{n} / \mathrm{a}$ & $\mathrm{n} / \mathrm{a}$ \\
\hline $\begin{array}{l}\text { Academic Progress Rate } \\
\text { [FTIC } 2 \text { year Retention Rate with GPA>2 ] }\end{array}$ & $\mathrm{n} / \mathrm{a}$ & $\mathrm{n} / \mathrm{a}$ & $\mathrm{n} / \mathrm{a}$ & $80 \%$ & $81 \%$ & $82 \%$ \\
\hline $\begin{array}{l}\text { University Access Rate } \\
\text { [Percent of Fall Undergraduates with a Pell grant] }\end{array}$ & $-14 \% \Delta$ & $42 \%$ & $42 \%$ & $42 \%$ & $42 \%$ & $42 \%$ \\
\hline $\begin{array}{l}\text { Bachelor's Degrees Awarded Within } \\
\text { Programs of Strategic Emphasis } \\
\text { [Based on list approved by BOG at 11/2013 meeting] }\end{array}$ & $18 \% \Delta$ & $40 \%$ & $47 \%$ & $47 \%$ & $49 \%$ & $49 \%$ \\
\hline $\begin{array}{l}\text { Graduate Degrees Awarded Within } \\
\text { Programs of Strategic Emphasis } \\
\text { [Based on list approved by BOG at 11/2013 meeting] }\end{array}$ & $-30 \% \Delta$ & $30 \%$ & $38 \%$ & $37 \%$ & $38 \%$ & $38 \%$ \\
\hline \multicolumn{7}{|l|}{ Board of Governors Choice Metric } \\
\hline $\begin{array}{l}\text { Percent of Bachelor's Degrees } \\
\text { Without Excess Hours }\end{array}$ & $17 \% \Delta$ & $70 \%$ & $71 \%$ & $72 \%$ & $73 \%$ & $75 \%$ \\
\hline \multicolumn{7}{|l|}{ Board of Trustees Choice Metric } \\
\hline Number of Postdoctoral Appointees & $\mathrm{n} / \mathrm{a}$ & $\mathrm{n} / \mathrm{a}$ & $\mathrm{n} / \mathrm{a}$ & $\mathrm{n} / \mathrm{a}$ & $\mathrm{n} / \mathrm{a}$ & $\mathrm{n} / \mathrm{a}$ \\
\hline
\end{tabular}

Note: Metrics are defined in appendix. 


\section{KEY PERFORMANCE INDICATORS}

The Board of Governors has selected the following Key Performance Indicators from its 2012-2025 System Strategic Plan and from accountability metrics identified by the Florida Legislature. The Key Performance Indicators emphasize three primary areas of focus: Academic Quality, Operational Efficiency, and Return on Investment. The indicators address common goals across all universities while also providing flexibility to address institution-specific goals from a list of metrics in the 2012-2025 System Strategic Plan.

The Goals Specific to Research Universities apply only to those universities classified by the Carnegie Foundation for the Advancement of Teaching as being a 'Research University'1, which includes Florida A\&M University (by university request), Florida Atlantic University, Florida International University, Florida State University, University of Central Florida, University of Florida, and the University of South Florida. 


\section{KEY PERFORMANCE INDICATORS}

The Board of Governors will consider the shaded 2014-15 goals for approval.

\section{Goals Common to All Universities}

\section{Academic Quality}

\section{National Ranking for University and Programs}

The USFSM College of Education's Center for Partnership in Arts-Integration was recently recognized by the Southern Regional Association of Teacher Education for its innovative programming. Additionally, the USFSM Hospitality Management program envisions a top ten ranking amongst all hospitality programs nationally within the next ten years.

\begin{tabular}{|c|c|c|c|c|c|c|}
\hline & $\begin{array}{l}\text { TREND } \\
\text { (2008-09 to } \\
2012-13)\end{array}$ & $\begin{array}{l}\text { 2012-13 } \\
\text { ACTUAL }\end{array}$ & $\begin{array}{c}\text { 2013-14 } \\
\text { ESTIMATES }\end{array}$ & $\begin{array}{l}2014-15 \\
\text { GOALS }\end{array}$ & $\begin{array}{l}\text { 2015-16 } \\
\text { GOALS }\end{array}$ & $\begin{array}{l}2016-17 \\
\text { GOALS }\end{array}$ \\
\hline SAT Score [for 3 subtests] & $\mathrm{n} / \mathrm{a}$ & $\mathrm{n} / \mathrm{a}$ & 1633 & 1,640 & 1,660 & 1,680 \\
\hline High School GPA & $\mathrm{n} / \mathrm{a}$ & $\mathrm{n} / \mathrm{a}$ & 3.6 & 3.65 & 3.7 & 3.75 \\
\hline $\begin{array}{l}\text { Professional/Licensure Exam } \\
\text { First-time Pass Rates }{ }^{1} \\
\text { Exams Above Benchmarks } \\
\text { Exams Below Benchmarks }\end{array}$ & $\begin{array}{l}\mathrm{n} / \mathrm{a} \\
\mathrm{n} / \mathrm{a}\end{array}$ & $\begin{array}{l}\mathrm{n} / \mathrm{a} \\
\mathrm{n} / \mathrm{a}\end{array}$ & $\begin{array}{l}\mathrm{n} / \mathrm{a} \\
\mathrm{n} / \mathrm{a}\end{array}$ & $\begin{array}{l}\mathrm{n} / \mathrm{a} \\
\mathrm{n} / \mathrm{a}\end{array}$ & $\begin{array}{l}\mathrm{n} / \mathrm{a} \\
\mathrm{n} / \mathrm{a}\end{array}$ & $\begin{array}{l}\mathrm{n} / \mathrm{a} \\
\mathrm{n} / \mathrm{a}\end{array}$ \\
\hline Operational Efficiency & & & & & & \\
\hline Freshman Retention Rate & $\mathrm{n} / \mathrm{a}$ & $\mathrm{n} / \mathrm{a}$ & $\mathrm{n} / \mathrm{a}$ & $77 \%$ & $80 \%$ & $83 \%$ \\
\hline $\begin{array}{l}\text { FTIC Graduation Rates } \\
\text { In } 4 \text { years (or less) } \\
\text { In } 6 \text { years (or less) }\end{array}$ & $\begin{array}{l}\mathrm{n} / \mathrm{a} \\
\mathrm{n} / \mathrm{a}\end{array}$ & $\begin{array}{l}\mathrm{n} / \mathrm{a} \\
\mathrm{n} / \mathrm{a}\end{array}$ & $\begin{array}{l}\mathrm{n} / \mathrm{a} \\
\mathrm{n} / \mathrm{a}\end{array}$ & $\begin{array}{l}\mathrm{n} / \mathrm{a} \\
\mathrm{n} / \mathrm{a}\end{array}$ & $\begin{array}{l}\mathrm{n} / \mathrm{a} \\
\mathrm{n} / \mathrm{a}\end{array}$ & $\begin{array}{l}42 \% \\
\mathrm{n} / \mathrm{a}\end{array}$ \\
\hline $\begin{array}{l}\text { AA Transfer Graduation Rates } \\
\text { In } 2 \text { years (or less) } \\
\text { In } 4 \text { years (or less) }\end{array}$ & $\begin{array}{l}3 \% \Delta \\
3 \% \Delta\end{array}$ & $\begin{array}{l}34 \% \\
65 \%\end{array}$ & $\begin{array}{l}37 \% \\
68 \%\end{array}$ & $\begin{array}{l}40 \% \\
71 \%\end{array}$ & $\begin{array}{l}43 \% \\
74 \%\end{array}$ & $\begin{array}{l}46 \% \\
77 \%\end{array}$ \\
\hline $\begin{array}{l}\text { Average Time to Degree } \\
\text { (for FTIC)* }\end{array}$ & $\mathrm{n} / \mathrm{a}$ & $\mathrm{n} / \mathrm{a}$ & $\mathrm{n} / \mathrm{a}$ & $\mathrm{n} / \mathrm{a}$ & $\mathrm{n} / \mathrm{a}$ & $4.3 \mathrm{yrs}$ \\
\hline Return on Investment & & & & & & \\
\hline Bachelor's Degrees Awarded & $24 \% \Delta$ & 556 & 490 & 505 & 520 & 535 \\
\hline $\begin{array}{l}\text { Percent of Bachelor's Degrees } \\
\text { in STEM }\end{array}$ & $-26 \% \Delta$ & $4 \%$ & $4 \%$ & $5 \%$ & $6 \%$ & $7 \%$ \\
\hline Graduate Degrees Awarded & $-52 \% \Delta$ & 60 & 49 & 51 & 52 & 54 \\
\hline $\begin{array}{l}\text { Percent of Graduate Degrees } \\
\text { in STEM }\end{array}$ & $\mathrm{n} / \mathrm{a}$ & $\mathrm{n} / \mathrm{a}$ & $\mathrm{n} / \mathrm{a}$ & $\mathrm{n} / \mathrm{a}$ & $\mathrm{n} / \mathrm{a}$ & $\mathrm{n} / \mathrm{a}$ \\
\hline Annual Gifts Received (\$M) & \multicolumn{6}{|c|}{ Reported at the USF System level } \\
\hline Endowment (\$M) & \multicolumn{6}{|c|}{ Reported at the USF System level } \\
\hline
\end{tabular}

Notes: (1) Professional licensure pass rates are based on the 2012-13 Annual Accountability Report with data that spans multiple time periods, (2) The methodology for calculating the percent of undergraduate seniors participating in a research course will be determined during the 2014 summer.

*Data produced by USFSM. 


\section{KEY PERFORMANCE INDICATORS}

\section{Institution Specific Goals}

Each university will provide updates for the metric goals reported in last year's Work Plans. The Board of Governors will consider the shaded 2014-15 goals for approval. University leadership will need to discuss any proposed changes with Board of Governors staff.

\begin{tabular}{|c|c|c|c|c|c|c|}
\hline & $\begin{array}{l}\text { TREND } \\
\text { (2008-09 to } \\
\text { 2012-13) }\end{array}$ & $\begin{array}{l}\text { 2012-13 } \\
\text { ACTUAL }\end{array}$ & $\begin{array}{c}2013-14 \\
\text { ESTIMATES }\end{array}$ & $\begin{array}{l}2014-15 \\
\text { GOALS }\end{array}$ & $\begin{array}{l}2015-16 \\
\text { GOALS }\end{array}$ & $\begin{array}{l}2016-17 \\
\text { GOALS }\end{array}$ \\
\hline $\begin{array}{l}\text { Metric \#1 Number of Adult (age } \\
25+\text { ) Undergraduates Enrolled } \\
\text { (in Fall) }\end{array}$ & $16 \%$ & 954 & 944 & 977 & 1,011 & 1,047 \\
\hline $\begin{array}{l}\text { Metric \#2 Percent of Course } \\
\text { Sections Offered via Distance and } \\
\text { Blended Learning }\end{array}$ & $55 \%$ & $43 \%$ & $45 \%$ & $46 \%$ & $47 \%$ & $47 \%$ \\
\hline $\begin{array}{l}\text { Metric \#3 Seek and/or Maintain } \\
\text { Carnegie's Community } \\
\text { Engagement classification }\end{array}$ & \multicolumn{6}{|c|}{$\begin{array}{l}\text { USF Sarasota-Manatee submitted an application to receive the Elective Community } \\
\text { Engagement Classification in April 2014. According to the Carnegie Foundation } \\
\text { timeline, designations will be announced in January } 2015 \text {. }\end{array}$} \\
\hline
\end{tabular}

To further distinguish the university's distinctive mission, the university may choose to provide two additional narrative and metric goals that are based on the university's own strategic plan.

Goal 1. Student Access: Expand access to University education that benefits students and the local, national, and global community.

\begin{tabular}{llllll|l} 
Metric: Students Served Explain $\quad 7 \% \Delta$ & 4,495 & 4,367 & 4,563 & 4,769 & 4,983
\end{tabular}

*Students served is an unduplicated, annual headcount of students, regardless of their home campus, enrolled in USFSM funded courses.

Goal 2. Student Success: Enhance success of student outcomes, faculty productivity, and community impact.

Metric: 4 year Success Rate of AA Transfer Students (retained or graduated from State University $-1 \% \Delta$ $80 \%$ $79 \%$ $80 \%$ $81 \%$ $82 \%$ System) 


\section{FISCAL INFORMATION}

University Revenues (in Millions of Dollars)

\begin{tabular}{|c|c|c|}
\hline & $\begin{array}{l}\text { 2013-14 } \\
\text { Actual }^{*}\end{array}$ & $\begin{array}{c}\text { 2014-15 } \\
\text { Appropriations }\end{array}$ \\
\hline \multicolumn{3}{|l|}{ Education \& General - Main Operations } \\
\hline State Funds & $\$ 13.1$ & $\$ 14.1$ \\
\hline Tuition & $\$ 8.9$ & $\$ 8.9$ \\
\hline TOTAL MAIN OPERATIONS & $\$ 22.0$ & $\$ 23.1$ \\
\hline \multicolumn{3}{|l|}{ Education \& General - Health-Science Center / Medical Schools } \\
\hline State Funds & $\mathrm{n} / \mathrm{a}$ & $\mathrm{n} / \mathrm{a}$ \\
\hline Tuition & $\mathrm{n} / \mathrm{a}$ & $\mathrm{n} / \mathrm{a}$ \\
\hline TOTAL HSC & $\mathrm{n} / \mathrm{a}$ & $\mathrm{n} / \mathrm{a}$ \\
\hline EDUCATION \& GENERAL TOTAL REVENUES & $\$ 22.0$ & $\$ 23.1$ \\
\hline
\end{tabular}

Note: State funds include General Revenue funds, Lottery funds, Federal Stimulus funds, and Phosphate Research funds (for Polytechnic) appropriated by the Florida Legislature (as reported in the Annual Accountability Report). Actual tuition includes base tuition and tuition differential fee revenues for resident and non-resident undergraduate and graduate students net of waivers (as reported in the Annual Accountability Report). Actual tuition revenues are not yet available for the 2013-14 year.

\section{OTHER BUDGET ENTITIES}

\section{Auxiliary Enterprises}

Resources associated with auxiliary units that are self supporting through fees, payments and charges. Examples include housing, food services, bookstores, parking services, health centers.

Revenues $\quad \$ 2.0 \quad n / a$

\section{Contracts \& Grants}

Resources received from federal, state or private sources for the purposes of conducting research and public service activities.

\begin{tabular}{lcc}
\hline \multicolumn{1}{c}{ Revenues } & $\$ 0.4$ & $\mathrm{n} / \mathrm{a}$ \\
\hline $\begin{array}{l}\text { Local Funds } \\
\text { Resources associated with student activity (supported by the student activity fee), student financial aid, concessions, intercollegiate } \\
\text { athletics, technology fee, green fee, and student life \& services fee. }\end{array}$ & $\mathrm{n} / \mathrm{a}$ \\
\hline \multicolumn{1}{l}{ Revenues } & $\$ 1.2$ & \\
\hline $\begin{array}{l}\text { Faculty Practice Plans } \\
\text { Revenues/receipts are funds generated from faculty practice plan activities. }\end{array}$ & $\begin{array}{c}\text { Reported at USF } \\
\text { System Level }\end{array}$ & $\mathrm{n} / \mathrm{a}$ \\
\hline \multicolumn{1}{l}{ Revenues } & $\$ 3.6$ & $\mathrm{n} / \mathrm{a}$ \\
\hline OTHER BUDGET ENTITY TOTAL REVENUES & $\$ 25.6$ & $\$ 23.1$ \\
\hline
\end{tabular}

*2013-14 estimated data is the 2013-14 operating budget. Ties to BOT approved FY2012-13 Annual Accountability Report. 


\section{FISCAL INFORMATION (continued)}

Undergraduate Resident Tuition Summary (for 30 credit hours)

\begin{tabular}{lccccc} 
& $\begin{array}{c}\text { FY 2012-13 } \\
\text { ACTUAL }\end{array}$ & $\begin{array}{c}\text { FY 2013-14 } \\
\text { ACTUAL }\end{array}$ & $\begin{array}{c}\text { FY 2014-15 } \\
\text { REQUEST }\end{array}$ & $\begin{array}{c}\text { FY 2015-16 } \\
\text { PLANNED }\end{array}$ & $\begin{array}{c}\text { FY 2016-17 } \\
\text { PLANNED }\end{array}$ \\
\hline Base Tuition & $\$ 3,100$ & $\$ 3,152$ & $\$ 3,152$ & $\$ 3,152$ & $\$ 3,152$ \\
\hline Tuition Differential Fee & $\$ 1,054$ & $\$ 1,054$ & $\$ 1,054$ & $\$ 1,054$ & $\$ 1,054$ \\
\hline Percent Increase $^{\text {Required Fees }}{ }^{1}$ & $11 \%$ & $1,3 \%$ & $0 \%$ & $0 \%$ & $0 \%$ \\
\hline TOTAL TUITION AND FEES $^{2}$ & $\$ 1,376$ & $\$ 1,381$ & $\$ 1,381$ & $\$ 1,381$ & $\$ 1,381$ \\
\hline
\end{tabular}

Note $^{1}$ : For more information regarding required fees see list of per credit hour fees and block fees on page 16.

\section{Student Debt Summary}

\begin{tabular}{lcccc|c} 
& $\begin{array}{c}2009-10 \\
\text { ACTUAL }\end{array}$ & $\begin{array}{c}2010-11 \\
\text { ACTUAL }\end{array}$ & $\begin{array}{c}2011-12 \\
\text { ACTUAL }\end{array}$ & $\begin{array}{c}2012-13 \\
\text { ACTUAL }\end{array}$ & $\begin{array}{c}2014-15 \\
\text { GOAL }\end{array}$ \\
\hline $\begin{array}{l}\text { Percent of Bachelor's Recipients with Debt } \\
\text { Average Amount of Debt }\end{array}$ & n/a & n/a & n/a & n/a & n/a \\
for Bachelor's who have graduated with debt & & n/a & n/a & n/a & n/a \\
NSLDS Cohort Year & 2008 & 2009 & 2010 & 2011 & 2012 \\
\hline Student Loan Cohort Default Rate (3rd Year) & $8.1 \%$ trial & $10.1 \%$ & $9.8 \%$ & $7.5 \%$ draft & $7.0 \%$
\end{tabular}

Cost of Attendance (for Full-Time Undergraduate Florida Residents in the Fall and Spring of 2013-14)

\begin{tabular}{cccccccc} 
& $\begin{array}{c}\text { TUITION } \\
\& \text { FEES }\end{array}$ & $\begin{array}{c}\text { BOOKS } \& \\
\text { SUPPLIES }\end{array}$ & $\begin{array}{c}\text { ROOM } \\
\text { \& BOARD }\end{array}$ & TRANSPORTATION & $\begin{array}{c}\text { OTHER } \\
\text { EXPENSES }\end{array}$ & TOTAL \\
\hline ON-CAMPUS & n/a & $n$ n/a & n/a & n/a & n/a & n/a \\
\hline AT HOME & $\$ 5,588$ & $\$ 1,000$ & $\$ 4,620$ & $\$ 1,600$ & $\$ 2,500$ & $\$ 15,308$ \\
\hline
\end{tabular}

${ }^{*}$ On-campus residence not available.

Estimated Net Cost by Family Income (for Full-Time Undergraduate Florida Residents in the Fall and Spring of 2013-14)

\begin{tabular}{|c|c|c|c|c|c|c|}
\hline \multirow{2}{*}{$\begin{array}{l}\text { FAMILY } \\
\text { INCOME } \\
\text { GROUPS }\end{array}$} & \multicolumn{2}{|c|}{$\begin{array}{l}\text { FULL-TIME RESIDENT } \\
\text { UNDERGRADUATES }\end{array}$} & \multirow{2}{*}{$\begin{array}{c}\text { AVG. NET } \\
\text { COST OF } \\
\text { ATTENDANCE }\end{array}$} & \multirow{2}{*}{$\begin{array}{l}\text { AVG. NET } \\
\text { TUITION } \\
\text { \& FEES } \\
\end{array}$} & \multirow{2}{*}{$\begin{array}{l}\text { AVERAGE } \\
\text { GIFT AID } \\
\text { AMOUNT }\end{array}$} & \multirow{2}{*}{$\begin{array}{l}\text { AVERAGE } \\
\text { LOAN } \\
\text { AMOUNT }\end{array}$} \\
\hline & HEADCOUNT & PERCENT & & & & \\
\hline Below $\$ 40,000$ & 250 & $47 \%$ & $\$ 12,395$ & $-\$ 1,337$ & $\$ 6,292$ & $\$ 5,099$ \\
\hline$\$ 40,000-\$ 59,999$ & 70 & $13 \%$ & $\$ 12,855$ & $-\$ 392$ & $\$ 5,409$ & $\$ 3,544$ \\
\hline$\$ 60,000-\$ 79,999$ & 43 & $8 \%$ & $\$ 15,311$ & $\$ 2,567$ & $\$ 2,411$ & $\$ 3,868$ \\
\hline$\$ 80,000-\$ 99,999$ & 31 & $6 \%$ & $\$ 15,128$ & $\$ 2,039$ & $\$ 2,907$ & $\$ 3,917$ \\
\hline$\$ 100,000$ Above & 70 & $13 \%$ & $\$ 15,579$ & $\$ 3,584$ & $\$ 1,504$ & $\$ 2,245$ \\
\hline Missing* & 65 & $13 \%$ & $\mathrm{n} / \mathrm{a}$ & $\$ 4,724$ & $\$ 292$ & $\$ 0$ \\
\hline TOTAL & 529 & $100 \%$ & $\$ 14,191$ & $\$ 699$ & $\$ 4,290$ & $\$ 3,720$ \\
\hline
\end{tabular}

Notes: This data only represents Fall and Spring financial aid data and is accurate as of March 31, 2014. Please note that small changes to Spring 2013 awards are possible before the data is finalized. Family Income Groups are based on the Total Family Income (including untaxed income) as reported on student FAFSA records. Full-time Students is a headcount based on at least 24 credit hours during Fall and Spring terms. Average Gift Aid includes all grants and scholarships from Federal, State, University and other private sources administered by the Financial Aid Office. Student waivers are also included in the Gift Aid amount. Gift Aid does not include the parental contribution towards EFC. Net Cost of Attendance is the actual average of the total Costs of Attendance (which will vary by income group due to the diversity of students living on- \& off- campus) minus the average Gift Aid amount. Net Tuition \& Fees is the actual average of the total costs of tuition and fees (which will vary by income group due to the amount of credit hours students are enrolled) minus the average Gift Aid amount (see page 16 for list of fees that are included). Average Loan Amount includes Federal (Perkins, Stafford, Ford Direct, and PLUS loans) and all private loans. The bottom-line Average represents the average of all full-time undergraduate Florida residents (note*: the total Net Cost of Attendance does not include students with missing family income data). 'Missing' includes students who did not file a FAFSA. 


\section{FISCAL INFORMATION (continued) \\ TUITION DIFFERENTIAL FEE INCREASE REQUEST FOR FALL 2014}

\section{Effective Date}

University Board of Trustees approval date:

\section{Campus or Center Location}

Campus or center location to which the tuition differential fee increase will apply (If the entire university, indicate as such):

\section{Undergraduate Course(s)}

Course(s). (If the tuition differential fee applies to all university undergraduate courses, indicate as such. If not, provide Tuition differential fee applies to all undergraduate courses.

rationale for the differentiation among courses):

\section{Current and Proposed Increase in the Tuition Differential Fee}

\begin{tabular}{l} 
Current Undergraduate Tuition Differential per credit hour: \\
\hline Percentage tuition differential fee increase (calculated as a \\
percentage of the sum of base tuition plus tuition differential):
\end{tabular}

$\$$ Increase in tuition differential per credit hour:

\$ Increase in tuition differential for 30 credit hours:

\section{Projected Differential Revenue Generated}

\begin{tabular}{l|l}
\hline Incremental revenue generated in 2014-15 (projected): & $\$ 0$
\end{tabular}

Total differential fee revenue generated in 2014-15 (projected): $\quad \$ 1,335,014$

\section{Intended Uses}

Describe how the revenue will be used.

\section{Describe the Impact to the Institution if Tuition Differential is Not Approved}

\section{Not applicable}

Request to Modify or Waive Tuition Differential Uses

(pursuant to Section 1001.706(3)(g) the Board may consider waiving its regulations associated with the $70 \% / 30 \%$ intended uses criteria identified in Regulation 7.001(14). If the university requests a modification; identify the modification, purpose of the modification, and rationale for the modification.) 


\section{FISCAL INFORMATION (continued) TUITION DIFFERENTIAL SUPPLEMENTAL INFORMATION}

Provide the following information for the 2013-14 academic year.

\begin{tabular}{|c|c|}
\hline $\begin{array}{c}2013-2014-70 \% \text { Initiatives (list the initiatives provided in } \\
\text { the } 2012-13 \text { tuition differential request) }\end{array}$ & University Update on Each Initiative \\
\hline Enhance timely college completion rates. & $\begin{array}{l}322 \text { courses funded. } \\
\text { 2-year graduation rates of AA transfers increased } 5 \% \text { over the } \\
\text { last year. } \\
\text { 4-year graduation rates of AA transfers increased } 12 \% \text { over } \\
\text { the last year. }\end{array}$ \\
\hline Additional Detail & where applicable: \\
\hline $\begin{array}{l}\text { Total Number of Faculty Hired or Retained (funded by tuition } \\
\text { differential): }\end{array}$ & 115 \\
\hline $\begin{array}{l}\text { Total Number of Advisors Hired or Retained (funded by } \\
\text { tuition differential): }\end{array}$ & 0 \\
\hline $\begin{array}{l}\text { Total Number of Course Sections Added or Saved (funded } \\
\text { by tuition differential): }\end{array}$ & 330 \\
\hline $\begin{array}{c}2013-2014-30 \% \text { Initiatives (list the initiatives provided in } \\
\text { the 2013-14 tuition differential request) }\end{array}$ & University Update on Each Initiative \\
\hline $\begin{array}{l}\text { More students supported with financial aid scholarships and } \\
\text { reduced loan indebtedness }\end{array}$ & $\begin{array}{l}\text { We increased the number of students receiving need based } \\
\text { grants from } 298 \text { in } 12-13 \text { to } 328 \text { this year, for an increase of } \\
10 \% \text {. }\end{array}$ \\
\hline $\begin{array}{l}\text { Provide financial support for talented students with limited } \\
\text { income. }\end{array}$ & $\begin{array}{l}\text { The total amount of grant aid for students increased by } 15 \% \\
\text { over } 2012-13 \text {. }\end{array}$ \\
\hline Additional Information (esti & ates as of February 28, 2014): \\
\hline $\begin{array}{l}\text { Unduplicated Count of Students Receiving at least one } \\
\text { Tuition Differential-Funded Award: }\end{array}$ & 328 \\
\hline $\begin{array}{l}\text { \$ Mean (per student receiving an award) of Tuition } \\
\text { Differential-Funded Awards: }\end{array}$ & $\$ 1,057$ \\
\hline $\begin{array}{l}\text { \$ Minimum (per student receiving an award) of Tuition } \\
\text { Differential-Funded Awards: }\end{array}$ & $\$ 120$ \\
\hline $\begin{array}{l}\text { \$ Maximum (per student receiving an award) of Tuition } \\
\text { Differential-Funded Awards: }\end{array}$ & $\$ 4,300$ \\
\hline
\end{tabular}




\section{FISCAL INFORMATION (continued)}

TUITION DIFFERENTIAL COLLECTIONS, EXPENDITURES, \& AVAILABLE BALANCES - FISCAL YEAR 2013-14 AND 2014-15

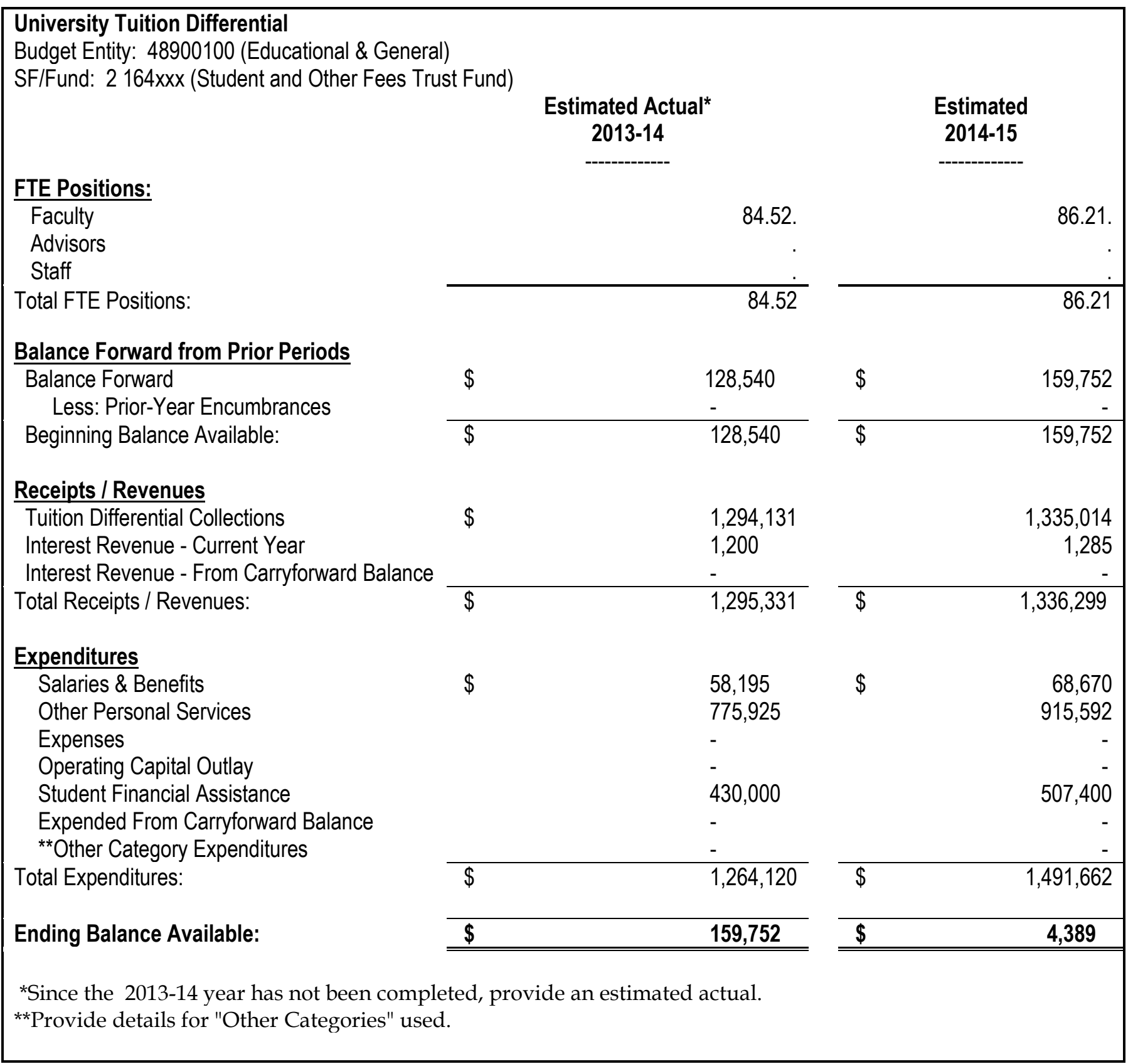




\section{FISCAL INFORMATION (continued) UNIVERSITY TUITION, FEES AND HOUSING PROJECTIONS}

\begin{tabular}{|c|c|c|c|c|c|c|c|}
\hline \multirow[t]{2}{*}{ Undergraduate Students } & \multicolumn{3}{|c|}{ - } & \multicolumn{4}{|c|}{ 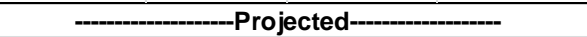 } \\
\hline & 2011-12 & 2012-13 & 2013-14 & 2014-15 & 2015-16 & 2016-17 & 2017-18 \\
\hline \multicolumn{8}{|l|}{ Tuition: } \\
\hline Base Tuition - (0\% inc. for $2014-15$ to $2017-18)$ & $\$ 103.32$ & $\$ 103.32$ & $\$ 105.07$ & $\$ 105.07$ & $\$ 105.07$ & $\$ 105.07$ & $\$ 105.07$ \\
\hline Tuition Differential & 21.42 & $\$ 35.14$ & $\$ 35.14$ & $\$ 35.14$ & $\$ 35.14$ & $\$ 35.14$ & $\$ 35.14$ \\
\hline Total Base Tuition \& Differential per Credit Hour & $\$ 124.74$ & $\$ 138.46$ & $\$ 140.21$ & $\$ 140.21$ & $\$ 140.21$ & $\$ 140.21$ & $\$ 140.21$ \\
\hline$\%$ Change & & $11.0 \%$ & $1.3 \%$ & $0.0 \%$ & $0.0 \%$ & $0.0 \%$ & $0.0 \%$ \\
\hline \multicolumn{8}{|l|}{ Fees (per credit hour): } \\
\hline Student Financial Aid ${ }^{1}$ & $\$ 5.16$ & $\$ 5.16$ & $\$ 5.25$ & $\$ 5.25$ & $\$ 5.25$ & $\$ 5.25$ & $\$ 5.25$ \\
\hline Capital Improvement $^{2}$ & $\$ 4.76$ & $\$ 6.76$ & $\$ 6.76$ & $\$ 6.76$ & $\$ 6.76$ & $\$ 6.76$ & $\$ 6.76$ \\
\hline Activity \& Service & $\$ 20.19$ & $\$ 20.19$ & $\$ 20.19$ & $\$ 20.19$ & $\$ 20.19$ & $\$ 20.19$ & $\$ 20.19$ \\
\hline Health & $\$ 4.03$ & $\$ 4.03$ & $\$ 4.03$ & $\$ 4.03$ & $\$ 4.03$ & $\$ 4.03$ & $\$ 4.03$ \\
\hline Athletic & $\$ 4.23$ & $\$ 4.23$ & $\$ 4.23$ & $\$ 4.23$ & $\$ 4.23$ & $\$ 4.23$ & $\$ 4.23$ \\
\hline \multicolumn{8}{|l|}{ Transportation Access } \\
\hline Technology ${ }^{1}$ & $\$ 5.16$ & $\$ 5.16$ & $\$ 5.25$ & $\$ 5.25$ & $\$ 5.25$ & $\$ 5.25$ & $\$ 5.25$ \\
\hline \multirow{2}{*}{\multicolumn{8}{|c|}{$\begin{array}{l}\text { Green Fee (USF, NCF, UWF only) } \\
\text { Student Life \& Services Fee (UNF only) }\end{array}$}} \\
\hline & & & & & & & \\
\hline \multicolumn{8}{|l|}{ Marshall Center Fee (USF only) } \\
\hline \multicolumn{8}{|l|}{ Student Affairs Facility Use Fee (FSU only) } \\
\hline Total Fees & $\$ 43.53$ & $\$ 45.53$ & $\$ 45.71$ & $\$ 45.71$ & $\$ 45.71$ & $\$ 45.71$ & $\$ 45.71$ \\
\hline Total Tuition and Fees per Credit Hour & $\$ 168.27$ & $\$ 183.99$ & $\$ 185.92$ & $\$ 185.92$ & $\$ 185.92$ & $\$ 185.92$ & $\$ 185.92$ \\
\hline$\%$ Change & & $9.3 \%$ & $1.0 \%$ & $0.0 \%$ & $0.0 \%$ & $0.0 \%$ & $0.0 \%$ \\
\hline \multicolumn{8}{|l|}{ Fees (block per term): } \\
\hline Activity \& Service & & & & & & & \\
\hline Health & & & & & & & \\
\hline Athletic & $\$ 5.00$ & $\$ 5.00$ & $\$ 5.00$ & $\$ 5.00$ & $\$ 5.00$ & $\$ 5.00$ & $\$ 5.00$ \\
\hline \multicolumn{8}{|l|}{ Transportation Access } \\
\hline \multicolumn{8}{|l|}{ Marshall Center Fee (USF only) } \\
\hline \multicolumn{8}{|l|}{ Student Affairs Facility Use Fee (FSU only) } \\
\hline \multicolumn{8}{|l|}{ List any new fee proposed } \\
\hline Total Block Fees per term & $\$ 5.00$ & $\$ 5.00$ & $\$ 5.00$ & $\$ 5.00$ & $\$ 5.00$ & $\$ 5.00$ & $\$ 5.00$ \\
\hline$\%$ Change & & $0.0 \%$ & $0.0 \%$ & $0.0 \%$ & $0.0 \%$ & $0.0 \%$ & $0.0 \%$ \\
\hline Total Tuition for 30 Credit Hours & $\$ 3,742.20$ & $\$ 4,153.80$ & $\$ 4,206.30$ & $\$ 4,206.30$ & $\$ 4,206.30$ & $\$ 4,206.30$ & $\$ 4,206.30$ \\
\hline Total Fees for 30 Credit Hours & $\$ 1,315.90$ & $\$ 1,375.90$ & $\$ 1,381.30$ & $\$ 1,381.30$ & $\$ 1,381.30$ & $\$ 1,381.30$ & $\$ 1,381.30$ \\
\hline Total Tuition and Fees for 30 Credit Hours & $\$ 5,058.10$ & $\$ 5,529.70$ & $\$ 5,587.60$ & $\$ 5,587.60$ & $\$ 5,587.60$ & $\$ 5,587.60$ & $\$ 5,587.60$ \\
\hline \$Change & & $\$ 471.60$ & $\$ 57.90$ & $\$ 0.00$ & $\$ 0.00$ & $\$ 0.00$ & $\$ 0.00$ \\
\hline$\%$ Change & & $9.3 \%$ & $1.0 \%$ & $0.0 \%$ & $0.0 \%$ & $0.0 \%$ & $0.0 \%$ \\
\hline \multicolumn{8}{|l|}{ Out-of-State Fees } \\
\hline Out-of-State Undergraduate Fee & $\$ 291.68$ & $\$ 315.00$ & $\$ 346.50$ & $\$ 346.50$ & $\$ 346.50$ & $\$ 346.50$ & $\$ 346.50$ \\
\hline Out-of-State Undergraduate Student Financial $\mathrm{Aid}^{3}$ & $\$ 14.58$ & $\$ 15.75$ & $\$ 22.57$ & $\$ 22.57$ & $\$ 22.57$ & $\$ 22.57$ & $\$ 22.57$ \\
\hline Total per credit hour & $\$ 306.26$ & $\$ 330.75$ & $\$ 369.07$ & $\$ 369.07$ & $\$ 369.07$ & $\$ 369.07$ & $\$ 369.07$ \\
\hline$\%$ Change & & $8.0 \%$ & $11.6 \%$ & $0.0 \%$ & $0.0 \%$ & $0.0 \%$ & $0.0 \%$ \\
\hline Total Tuition for 30 Credit Hours & $\$ 12,492.60$ & $\$ 13,603.80$ & $\$ 14,601.30$ & $\$ 14,601.30$ & $\$ 14,601.30$ & $\$ 14,601.30$ & $\$ 14,601.30$ \\
\hline Total Fees for $\mathbf{3 0}$ Credit Hours & $\$ 1,753.30$ & $\$ 1,848.40$ & $\$ 2,058.40$ & $\$ 2,058.40$ & $\$ 2,058.40$ & $\$ 2,058.40$ & $\$ 2,058.40$ \\
\hline Total Tuition and Fees for 30 Credit Hours & $\$ 14,245.90$ & $\$ 15,452.20$ & $\$ 16,659.70$ & $\$ 16,659.70$ & $\$ 16,659.70$ & $\$ 16,659.70$ & $\$ 16,659.70$ \\
\hline \$Change & & $\$ 1,206.30$ & $\$ 1,207.50$ & $\$ 0.00$ & $\$ 0.00$ & $\$ 0.00$ & $\$ 0.00$ \\
\hline$\%$ Change & & $8.5 \%$ & $7.8 \%$ & $0.0 \%$ & $0.0 \%$ & $0.0 \%$ & $0.0 \%$ \\
\hline & & & & & & & \\
\hline${ }^{1}$ can be no more than $5 \%$ of tuition. & ${ }^{3}$ can be no more & than $5 \%$ of tuition & and the out-of-sta & & & & \\
\hline 2 as approved by the Board of Governors. & ${ }^{4}$ combine the $\mathrm{mo}$ & it popular housin & and dining plans & vided to students & & & \\
\hline & & & & & & & \\
\hline
\end{tabular}




\section{ENROLLMENT PLANNING}

Planned Enrollment Growth by Student Type (for all E\&G students at all campuses)

\begin{tabular}{|c|c|c|c|c|c|c|c|c|c|}
\hline \multirow{2}{*}{ UNDERGRADUATE } & \multirow[t]{2}{*}{$\begin{array}{l}5 \text { YEAR } \\
\text { TREND } \\
(2008-13)\end{array}$} & \multicolumn{2}{|c|}{$\begin{array}{c}\text { Fall } 2013 \\
\text { ACTUAL } \\
\text { HEADCOUNT }\end{array}$} & \multicolumn{2}{|c|}{$\begin{array}{c}\text { Fall } 2014 \\
\text { PLANNED } \\
\text { HEADCOUNT }\end{array}$} & \multicolumn{2}{|c|}{$\begin{array}{c}\text { Fall } 2015 \\
\text { PLANNED } \\
\text { HEADCOUNT }\end{array}$} & \multicolumn{2}{|c|}{$\begin{array}{c}\text { Fall } 2016 \\
\text { PLANNED } \\
\text { HEADCOUNT }\end{array}$} \\
\hline & & & & & & & & & \\
\hline FTIC (Regular Admit) & $\mathrm{n} / \mathrm{a}$ & 86 & $5 \%$ & 230 & $13 \%$ & 362 & $18 \%$ & 495 & $22 \%$ \\
\hline FTIC (Profile Admit) & $\mathrm{n} / \mathrm{a}$ & 0 & $0 \%$ & 5 & $0 \%$ & 8 & $0 \%$ & 10 & $0 \%$ \\
\hline AA Transfers* & $-2 \% \Delta$ & 1,058 & $63 \%$ & 1,095 & $58 \%$ & 1,133 & $55 \%$ & 1,173 & $52 \%$ \\
\hline Other Transfers & $18 \% \Delta$ & 527 & $32 \%$ & 545 & $29 \%$ & 565 & $27 \%$ & 584 & $26 \%$ \\
\hline Subtotal & $10 \% \Delta$ & 1,671 & $100 \%$ & 1,875 & $100 \%$ & 2,068 & $100 \%$ & 2,261 & $100 \%$ \\
\hline \multicolumn{10}{|l|}{ GRADUATE STUDENTS } \\
\hline Master's & $-63 \% \Delta$ & 128 & $100 \%$ & 134 & $100 \%$ & 140 & $100 \%$ & 146 & $100 \%$ \\
\hline Research Doctoral & $\mathrm{n} / \mathrm{a}$ & 0 & $0 \%$ & 0 & $0 \%$ & 0 & $0 \%$ & 0 & $0 \%$ \\
\hline Professional Doctoral & $\mathrm{n} / \mathrm{a}$ & 0 & $0 \%$ & 0 & $0 \%$ & 0 & $0 \%$ & 0 & $0 \%$ \\
\hline Subtotal & $-63 \% \Delta$ & 128 & $100 \%$ & 134 & $100 \%$ & 140 & $100 \%$ & 146 & $100 \%$ \\
\hline NOT-DEGREE SEEKING & $-38 \% \Delta$ & 88 & & 91 & & 94 & & 98 & \\
\hline MEDICAL & $n / a$ & 0 & & 0 & & 0 & & 0 & \\
\hline TOTAL & $-6 \% \Delta$ & 1,886 & & 2,100 & & 2,302 & & 2,506 & \\
\hline
\end{tabular}

Note $^{*}$ AA transfers refer only to transfers from the Florida College System.

Planned Enrollment Growth by Method of Instruction (for all E\&G students at all campuses)

\begin{tabular}{|c|c|c|c|c|c|c|c|c|c|}
\hline & \multirow{2}{*}{$\begin{array}{c}2 \text { YEAR } \\
\text { TREND } \\
\text { (2010-11 to } \\
2012-13)\end{array}$} & \multicolumn{2}{|c|}{$2012-13$} & \multicolumn{2}{|c|}{ 2014-15 } & \multicolumn{2}{|c|}{$2015-16$} & \multicolumn{2}{|c|}{ 2016-17 } \\
\hline & & $\begin{array}{l}\text { ACTUAL } \\
\text { FTE }\end{array}$ & $\begin{array}{c}\% \text { of } \\
\text { TOTAL }\end{array}$ & $\begin{array}{l}\text { PLANNED } \\
\text { FTE }\end{array}$ & $\begin{array}{c}\% \text { of } \\
\text { TOTAL }\end{array}$ & $\begin{array}{l}\text { PLANNED } \\
\text { FTE }\end{array}$ & $\begin{array}{c}\% \text { of } \\
\text { TOTAL }\end{array}$ & $\begin{array}{l}\text { PLANNED } \\
\text { FTE }\end{array}$ & $\begin{array}{l}\% \text { of } \\
\text { TOTAL }\end{array}$ \\
\hline \multicolumn{10}{|l|}{ UNDERGRADUATE } \\
\hline DISTANCE $(>80 \%)$ & $14 \%$ & 533 & $49 \%$ & 556 & $50 \%$ & 581 & $50 \%$ & 607 & $50 \%$ \\
\hline HYBRID (50\%-79\%) & $24 \%$ & 33 & $3 \%$ & 44 & $4 \%$ & 46 & $4 \%$ & 48 & $4 \%$ \\
\hline TRADITIONAL (<50\%) & $-9 \%$ & 525 & $48 \%$ & 511 & $46 \%$ & 534 & $46 \%$ & 559 & $46 \%$ \\
\hline TOTAL & $2 \%$ & 1,091 & $100 \%$ & 1,111 & $100 \%$ & 1,161 & $100 \%$ & 1,214 & $100 \%$ \\
\hline \multicolumn{10}{|l|}{ GRADUATE } \\
\hline DISTANCE $(80 \%)$ & $-10 \%$ & 21 & $22 \%$ & 23 & $23 \%$ & 25 & $23 \%$ & 26 & $23 \%$ \\
\hline HYBRID $(50 \%-79 \%)$ & $48 \%$ & 4 & $4 \%$ & 4 & $4 \%$ & 4 & $4 \%$ & 5 & $4 \%$ \\
\hline TRADITIONAL (<50\%) & $-28 \%$ & 72 & $74 \%$ & 75 & $73 \%$ & 78 & $73 \%$ & 81 & $73 \%$ \\
\hline TOTAL & $-23 \%$ & 97 & $100 \%$ & 102 & $100 \%$ & 107 & $100 \%$ & 112 & $100 \%$ \\
\hline
\end{tabular}

Note: Full-time Equivalent (FTE) student is a measure of instructional effort (and student activity) that is based on the number of credit hours that students enroll. FTE is based on the Florida definition, which divides undergraduate credit hours by 40 and graduate credit hours by 32 . Distance Learning is a course in which at least 80 percent of the direct instruction of the course is delivered using some form of technology when the student and instructor are separated by time or space, or both (per 1009.24(17), F.S.). Hybrid is a course where $50 \%$ to $79 \%$ of the instruction is delivered using some form of technology, when the student and instructor are separated by time or space, or both (per SUDS data element 2052). Traditional (and Technology

Enhanced) refers to primarily face to face instruction utilizing some form of technology for delivery of supplemental course materials for no more than $49 \%$ of instruction (per SUDS data element 2052). 


\section{ENROLLMENT PLANNING (continued)}

\section{Planned Enrollment Plan by Residency and Student Level (Florida FTE)}

\begin{tabular}{|c|c|c|c|c|c|c|c|c|c|}
\hline & $\begin{array}{c}\text { Estimated } \\
\text { Actual } \\
\text { 2013-14 }\end{array}$ & $\begin{array}{c}\text { Funded } \\
2014-15 \\
\end{array}$ & $\begin{array}{c}\text { Planned } \\
2014-15 \\
\end{array}$ & $\begin{array}{c}\text { Planned } \\
2015-16 \\
\end{array}$ & $\begin{array}{c}\text { Planned } \\
2016-17 \\
\end{array}$ & $\begin{array}{c}\text { Planned } \\
2017-18 \\
\end{array}$ & $\begin{array}{c}\text { Planned } \\
2018-19 \\
\end{array}$ & $\begin{array}{c}\text { Planned } \\
2019-20 \\
\end{array}$ & $\begin{array}{c}\text { Planned } \\
\text { Annual } \\
\text { Growth } \\
\text { Rate* }^{*}\end{array}$ \\
\hline \multicolumn{10}{|c|}{ STATE FUNDABLE } \\
\hline \multicolumn{10}{|c|}{ Florida Resident } \\
\hline LOWER & 205 & 0 & 214 & 224 & 234 & 244 & 255 & 267 & $4.92 \%$ \\
\hline UPPER & 820 & 798 & 857 & 896 & 936 & 978 & 1,022 & 1,069 & $4.92 \%$ \\
\hline GRAD I & 93 & 182 & 97 & 101 & 106 & 111 & 116 & 121 & $4.92 \%$ \\
\hline GRAD ॥ & 0 & 0 & 0 & 0 & 0 & 0 & 0 & 0 & $4.92 \%$ \\
\hline TOTAL & 1,118 & 980 & 1,169 & 1,221 & 1,276 & 1,333 & 1,393 & 1,456 & $4.92 \%$ \\
\hline \multicolumn{10}{|c|}{ Non- Resident } \\
\hline LOWER & 12 & $\mathrm{n} / \mathrm{a}$ & 13 & 13 & 14 & 15 & 15 & 16 & $4.92 \%$ \\
\hline UPPER & 26 & $\mathrm{n} / \mathrm{a}$ & 27 & 28 & 29 & 31 & 32 & 34 & $4.92 \%$ \\
\hline GRAD I & 5 & $\mathrm{n} / \mathrm{a}$ & 5 & 6 & 6 & 6 & 6 & 7 & $4.92 \%$ \\
\hline GRAD ॥ & 0 & $\mathrm{n} / \mathrm{a}$ & 0 & 0 & 0 & 0 & 0 & 0 & $\mathrm{n} / \mathrm{a}$ \\
\hline TOTAL & 43 & $\mathrm{n} / \mathrm{a}$ & 45 & 47 & 49 & 52 & 54 & 56 & $4.92 \%$ \\
\hline \multicolumn{10}{|l|}{ TOTAL } \\
\hline LOWER & 217 & n/a & 227 & 237 & 248 & 259 & 271 & 283 & $4.92 \%$ \\
\hline UPPER & 846 & $\mathrm{n} / \mathrm{a}$ & 884 & 924 & 966 & 1,009 & 1,055 & 1,102 & $4.92 \%$ \\
\hline GRAD I & 98 & $\mathrm{n} / \mathrm{a}$ & 102 & 107 & 112 & 117 & 122 & 128 & $4.92 \%$ \\
\hline GRAD ॥ & 0 & $\mathrm{n} / \mathrm{a}$ & 0 & 0 & 0 & 0 & 0 & 0 & $4.92 \%$ \\
\hline TOTAL & 1161 & $\mathrm{n} / \mathrm{a}$ & 1,213 & 1,268 & 1,325 & 1,385 & 1,447 & 1,513 & $4.92 \%$ \\
\hline \multicolumn{10}{|c|}{ NOT STATE FUNDABLE } \\
\hline LOWER & 4 & $\mathrm{n} / \mathrm{a}$ & 5 & 5 & 5 & 5 & 6 & 6 & $4.92 \%$ \\
\hline UPPER & 15 & $\mathrm{n} / \mathrm{a}$ & 16 & 17 & 18 & 18 & 19 & 20 & $4.92 \%$ \\
\hline GRAD I & 3 & $\mathrm{n} / \mathrm{a}$ & 3 & 3 & 3 & 3 & 3 & 4 & $4.92 \%$ \\
\hline GRAD ॥ & 0 & n/a & 0 & 0 & 0 & 0 & 0 & 0 & \\
\hline TOTAL & 23 & n/a & 24 & 25 & 26 & 27 & 28 & 29 & $4.92 \%$ \\
\hline
\end{tabular}

Note: Full-time Equivalent (FTE) student is a measure of instructional effort (and student activity) that is based on the number of credit hours that students enroll. FTE is based on the Florida definition, which divides undergraduate credit hours by 40 and graduate credit hours by 32 . Note*:The average annual growth rate is based on the annual growth rate from 2014-15 to 2019-20. 


\section{ACADEMIC PROGRAM COORDINATION}

New Programs For Consideration by University in AY 2014-15

The S.U.S. Council of Academic Vice Presidents (CAVP) Academic Program Coordination Work Group will review these programs as part of their on-going coordination efforts. The programs listed below are based on the 2013-14 Work Plan list for programs under consideration for 2014-16.

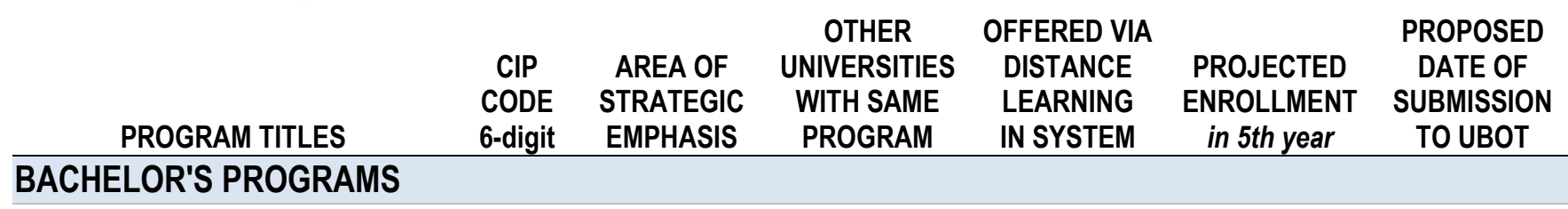

\section{MASTER'S, SPECIALIST AND OTHER ADVANCED MASTER'S PROGRAMS}

\section{DOCTORAL PROGRAMS}

New Programs For Consideration by University in 2015-17

These programs will be used in the 2015-16 Work Plan list for programs under consideration for 2015-16.

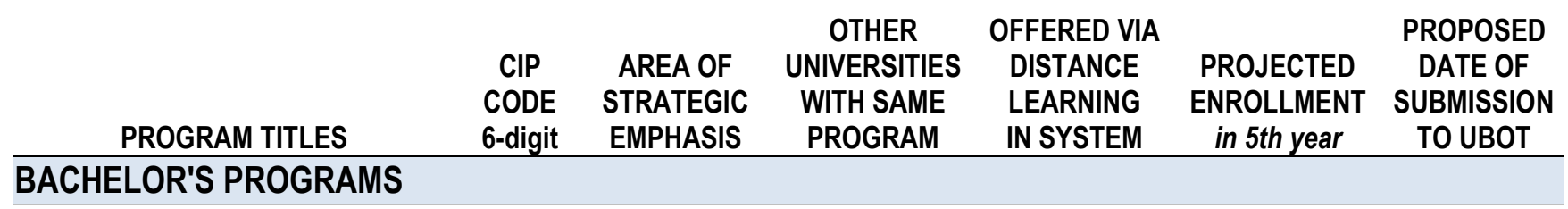

\section{MASTER'S, SPECIALIST AND OTHER ADVANCED MASTER'S PROGRAMS}




\section{DEFINITIONS}

\section{Performance Based Funding}

Percent of Bachelor's Graduates Employed Fulltime in Florida or Continuing their Education in the U.S. One Year After Graduation

\section{Median Wages of Bachelor's Graduates Employed Full-time in Florida One Year After Graduation}

\section{Average Cost per Bachelor's Degree} Instructional costs to the university
This metric is based on the percentage of a graduating class of bachelor's degree recipients who are employed full-time in Florida or continuing their education somewhere in the United States. Students who do not have valid social security numbers are excluded.

Note: Board staff have been in discussions with the Department of Economic Opportunity staff about the possibility of adding non-Florida employment data (from Wage Record Interchange System (WRIS2) to this metric for future evaluation.

Sources: State University Database System (SUDS), Florida Education \& Training Placement Information Program (FETPIP), National Student Clearinghouse.
This metric is based on annualized Unemployment Insurance (UI) wage data from the fourth fiscal quarter after graduation for bachelor's recipients. UI wage data does not include individuals who are self-employed, employed out of state, employed by the military or federal government, those without a valid social security number, or making less than minimum wage. Sources: State University Database System (SUDS), Florida Education \& Training Placement Information Program (FETPIP), National Student Clearinghouse.

For each of the last four years of data, the annual total undergraduate instructional expenditures were divided by the total fundable student credit hours to create a cost per credit hour for each year. This cost per credit hour was then multiplied by 30 credit hours to derive an average annual cost. The average annual cost for each of the four years was summed to provide an average cost per degree for a baccalaureate degree that requires 120 credit hours. Sources: State University Database System (SUDS), Expenditure Analysis: Report IV (2009-10 through 2012-13).

This metric is based on the percentage of first-time-in-college (FTIC) students who started in

Six Year FTIC Graduation Rate

\section{Academic} Progress Rate 2nd Year Retention with GPA Above 2.0

University Access Rate Percent of Undergraduates with a Pell-grant

\section{Bachelor's} Degrees Awarded within Programs of Strategic Emphasis (includes STEM)

\section{Graduate} Degrees Awarded within Programs of Strategic Emphasis (includes STEM) the Fall (or summer continuing to Fall) term and had graduated from the same institution within six years. Students of degree programs longer than four years (eg, PharmD) are included in the cohorts. Students who are active duty military are not included in the data. Source: State University Database System (SUDS).

This metric is based on the percentage of first-time-in-college (FTIC) students who started in the Fall (or summer continuing to Fall) term and were enrolled full-time in their first semester and were still enrolled in the same institution during the Fall term following their first year with had a grade point average (GPA) of at least 2.0 at the end of their first year (Fall, Spring, Summer).

Source: State University Database System (SUDS).

This metric is based the number of undergraduates, enrolled during the fall term, who received a Pell-grant during the fall term. Unclassified students, who are not eligible for Pell-grants, were excluded from this metric. Source: State University Database System (SUDS).

This metric is based on the number of baccalaureate degrees awarded within the programs designated by the Board of Governors as 'Programs of Strategic Emphasis'. A student who has multiple majors in the subset of targeted Classification of Instruction Program codes will be counted twice (i.e., double-majors are included).

Source: State University Database System (SUDS).

This metric is based on the number of graduate degrees awarded within the programs designated by the Board of Governors as 'Programs of Strategic Emphasis'. A student who has multiple majors in the subset of targeted Classification of Instruction Program codes will be counted twice (i.e., double-majors are included).

Source: State University Database System (SUDS). 
Freshmen in Top $10 \%$ of High School Class Applies to: NCF

\section{BOG Choice Metrics}

Percent of all degree-seeking, first-time, first-year (freshman) students who had high school class rank within the top $10 \%$ of their graduating high school class.

Source: New College of Florida.

\section{Percent of Bachelor's Degrees Without Excess Hours}

This metric is based on the percentage of baccalaureate degrees awarded within $110 \%$ of the credit hours required for a degree based on the Board of Governors Academic Program Inventory.

Note: It is important to note that the statutory provisions of the "Excess Hour Surcharge" $(1009.286, F S)$ have been modified several times by the Florida Legislature, resulting in a phased-in approach that has created three different cohorts of students with different requirements. The performance funding metric data is based on the latest statutory requirements that mandates $110 \%$ of required hours as the threshold. In accordance with statute, this metric excludes the following types of student credits (ie, accelerated mechanisms, remedial coursework, non-native credit hours that are not used toward the degree, non-native credit hours from failed, incomplete, withdrawn, or repeated courses, credit hours from internship programs, credit hours up to 10 foreign language credit hours for transfer students in Florida, and credit hours earned in military science courses that are part of the Reserve Officers' Training Corps (ROTC) program).

Source: State University Database System (SUDS).

This metric is based on the number of awards that faculty have earned in the arts, humanities, science, engineering and health fields as reported in the annual 'Top American Research Universities' report. Twenty-three of the most prominent awards are considered, including: Getty Scholars in Residence, Guggenheim Fellows, Howard Hughes Medical Institute Investigators, MacArthur Foundation Fellows, National Endowment for the Humanities (NEH) Fellows, National Medal of Science and National Medal of Technology, Robert Wood Johnson Policy Fellows, Sloan Research Fellows, Woodrow Wilson Fellows, to name a few awards. Source: Center for Measuring University Performance, Annual Report of the Top American Research Universities (TARU).

This metric is based on the number of Top 50 university rankings that NCF earned from the following list of publications: US News and World Report, Forbes, Kiplinger, Washington Monthly, Center for Measuring University Performance, Times Higher Education World University Rankings, QS World University Ranking, and the Academic Ranking of World Universities.

Source: Board of Governors staff review.

\section{National Ranking for Institutional \& Program Achievements}

\section{BOT Choice Metrics}

Percent of R\&D Expenditures Funded from External Sources FAMU

\section{Bachelor's Degrees Awarded to Minorities FAU, FGCU, FIU}

This metric reports the amount of research expenditures that was funded from federal, private industry and other (non-state and non-institutional) sources.

Source: National Science Foundation annual survey of Higher Education Research and Development (HERD).

This metric is the number, or percentage, of baccalaureate degrees granted in an academic year to Non-Hispanic Black and Hispanic students. This metric does not include students classified as Non-Resident Alien or students with a missing race code. Source: State University Database System (SUDS).

This metric is based on the difference between the Financial Resources rank and the overall University rank. U.S. News measures financial resources by using a two-year average spending per student on instruction, research, student services and related educational expenditures spending on sports, dorms and hospitals doesn't count. Source: US News and World Report's annual National University rankings. 


\section{Percent of Undergraduate Seniors Participating in a Research Course NCF}

\section{Number of Bachelor Degrees Awarded Annually UCF}

\section{Total Research Expenditures UF}

Percent of Course Sections
Offered via Distance and
Blended Learning
UNF

This metric is based on the percentage of undergraduate seniors who participate in a research course during their senior year.

Source: New College of Florida.

This metric is the number of baccalaureate degrees granted in an academic year. Students who earned two distinct degrees in the same academic year were counted twice; students who completed multiple majors or tracks were only counted once.

Source: State University Database System (SUDS).

This metric is the total expenditures (includes non-science \& engineering fields) for research \& development activities within a given fiscal year.

Source: National Science Foundation annual survey of Higher Education Research and Development (HERD).

This metric is based on the percentage of course sections classified as having at least $50 \%$ of the instruction delivered using some form of technology, when the student and instructor are separated by time or space, or both. Source: State University Database System (SUDS).

This metric is based on the number of post-doctoral appointees at the beginning of the academic year. A postdoctoral researcher has recently earned a doctoral (or foreign equivalent) degree and has a temporary paid appointment to focus on specialized research/scholarship under the supervision of a senior scholar.

Source: National Science Foundation/National Institutes of Health annual Survey of Graduate Students and Postdoctorates in Science and Engineering (GSS).

\section{Percentage of Adult Undergraduates Enrolled UWF}

This metric is based on the percentage of undergraduates (enrolled during the fall term) who are at least 25 years old at the time of admission. This includes undergraduates who are not degree-seeking, or unclassified.

Source: State University Database System (SUDS).

\section{Preeminent Research University Funding Metrics}

\section{Average GPA and SAT Score}

\section{Public University National Ranking}

Freshman Retention Rate (Full-time, FTIC)

6-year Graduation Rate (Full-time, FTIC)
An average weighted grade point average of 4.0 or higher and an average SAT score of 1800 or higher for fall semester incoming freshmen, as reported annually in the admissions data that universities submit to the Board of Governors. This data includes registered FTIC (student type='B','E') with an admission action of admitted or provisionally admitted ('A','P','X').

A top-50 ranking on at least two well-known and highly respected national public university rankings, reflecting national preeminence, using most recent rankings. Legislative staff based their initial evaluation on the following list: US News and World Report, Forbes, Kiplinger, Washington Monthly, Center for Measuring University Performance, Times Higher Education World University Rankings, QS World University Ranking, and the Academic Ranking of World Universities.

Freshman Retention Rate (Full-time, FTIC) as reported annually to the Integrated Postsecondary Education Data System (IPEDS). The retention rates that are reported in the Board's annual Accountability report are preliminary because they are based on student enrollment in their second fall term as reported by the 28th calendar day following the first day of class. When the Board of Governors reports final retention rates to IPEDS in the Spring (usually the first week of April), that data is based on the student enrollment data as reported after the Fall semester has been completed. The preliminary and final retention rates are nearly identical when rounded to the nearest whole number.

6-year Graduation Rate (Full-time, FTIC) as reported annually to the Integrated Postsecondary Education Data System (IPEDS). The Board of Governors reports the preliminary graduation rates in the annual Accountability report, and 'final' graduation rates to IPEDS in the beginning of February. The final rates are usually the same as the preliminary rates but can be slightly higher ( $1 \%-2 \%$ points) due to cohort adjustments for specific, and rare, exemptions allowed by IPEDS. 


\section{National Academy \\ Memberships}

Total Annual Research Expenditures (\$M) (Science \& Engineering only)

Total Annual Research Expenditures in Diversified Non-Medical Sciences (\$M) (Science \& Engineering only)

National Ranking in S.T.E.M. Research Expenditures

\section{Patents Awarded}

(over 3 year period)

Doctoral Degrees Awarded Annually

Number of Post-Doctoral
Appointees

Endowment Size (\$M)
National Academy Memberships held by faculty as reported by the Center for Measuring University Performance in the Top American Research Universities (TARU) annual report.

Total Science \& Engineering Research Expenditures, including federal research expenditures, of $\$ 200$ million or more, as reported annually by the National Science Foundation (NSF).

Total S\&E research expenditures in non-medical sciences as reported by the NSF. This removes medical sciences funds ( $9 F$ \& $12 F$ in HERD survey) from the total S\&E amount.

The NSF identifies 8 broad disciplines within Science \& Engineering (Computer Science, Engineering, Environmental Science, Life Science, Mathematical Sciences, Physical Sciences, Psychology, Social Sciences). The rankings by discipline are determined by BOG staff using the NSF WebCaspar database.

Total patents awarded by the United States Patent and Trademark Office (USPTO) for the most recent 3-year period. Due to a year-lag in published reports, Board of Governors staff query the USPTO database with a query that only counts utility patents:"(AN/"University Name" AND ISD/20100101->20131231 AND APT/1)".

Doctoral degrees awarded annually, as reported annually in the Board of Governors Accountability Report. Note: per legislative workpapers, this metric does not include Professional degrees.

The number of Postdoctoral Appointees awarded annually, as reported in the TARU annual report. This data is based on National Science Foundation/National Institutes of Health annual Survey of Graduate Students and Postdoctorates in Science and Engineering (GSS).

This data comes from the National Association of College and University Business Officers (NACUBO) and Commonfund Institute's annual report of Market Value of Endowment Assets which, due to timing, may release the next fiscal year's data after the Board of Governors Accountability report is published. 


\section{Goals Common to All Universities}

Academic Quality

Avg. SAT Score (for 3 subtests)

An average weighted grade point average of 4.0 or higher and an average SAT score of 1800 or higher for fall semester incoming freshmen, as reported annually in the admissions data that universities submit to the Board of Governors. This data includes registered FTIC (student type='B','E') with an admission action of admitted or provisionally admitted ('A','P','X').

Avg. HS GPA

Professional/Licensure Exam First-time Pass Rates

Operational Efficiency

Freshman Retention Rate

FTIC Graduation Rates In 4 years (or less)

In 6 years (or less)

\section{AA Transfer Graduation Rates \\ In 2 years (or less) \\ In 4 years (or less)}

\section{Average Time to Degree (for FTIC)}

\section{Return on Investment}

Bachelor's Degrees Awarded

Percent of Bachelor's

Degrees in STEM

Graduate Degrees Awarded

Percent of Graduate Degrees in STEM

Annual Gifts Received (\$M)

Endowment $(\$ M)$
The average HS GPA for Admitted \& Registered FTIC and early admit (B,E) students. Max score is 5.0.

The number of exams with first-time pass rates above and below the national or state average, as reported in the 2012-13 Accountability report, including: Nursing, Law, Medicine (3 subtests), Veterinary, Pharmacy, Dental (2 subtests), Physical Therapy, and Occupational Therapy.

The percentage of a full-time, first-time-in-college (FTIC) undergraduate cohort (entering in fall term or summer continuing to fall) that is still enrolled or has graduated from the same institution in the following fall term as reported in the 2012-13 Accountability report (table 4B) - see link.

As reported in the 2012-13 Accountability report (table 4D), First-time-in-college (FTIC) cohort is defined as undergraduates entering in fall term (or summer continuing to fall) with fewer than 12 hours earned since high school graduation. The rate is the percentage of the initial cohort that has either graduated from or is still enrolled in the same institution by the fourth or sixth academic year. Both full-time and part-time students are used in the calculation. The initial cohort is revised to remove students, who have allowable exclusions as defined by IPEDS, from the cohort.

As reported in the 2012-13 Accountability report (table 4E), AA Transfer cohort is defined as undergraduates entering in the fall term (or summer continuing to fall) and having earned an $A A$ degree from an institution in the Florida College System. The rate is the percentage of the initial cohort that has either graduated from or is still enrolled in the same institution by the second or fourth academic year. Both full-time and part-time students are used in the calculation. The initial cohort is revised to remove students, who have allowable exclusions as defined by IPEDS, from the cohort.

This metric is the number of years between the start date (using date of most recent admission) and the end date (using the last month in the term degree was granted) for a graduating class of first-time, single-major baccalaureates in 120 credit hour programs within a (Summer, Fall, Spring) year.

This is a count of baccalaureate degrees awarded as reported in the 2012-13 Accountability Report (table 4G).

The percentage of baccalaureate degrees that are classified as STEM by the Board of Governors in the SUS program inventory as reported in the 2012-13 Accountability Report (table 4H).

This is a count of graduate degrees awarded as reported in the 2012-13 Accountability Report (table 5B).

The percentage of baccalaureate degrees that are classified as STEM by the Board of Governors in the SUS program inventory as reported in the 2012-13 Accountability Report (table 5C).

As reported in the Council for Aid to Education's Voluntary Support of Education (VSE) survey in the section entitled "Gift Income Summary," this is the sum of the present value of all gifts (including outright and deferred gifts) received for any purpose and from all sources during the fiscal year, excluding pledges and bequests. (There's a deferred gift calculator at www.cae.org/vse.) The present value of non-cash gifts is defined as the tax deduction to the donor as allowed by the IRS.

Endowment value at the end of the fiscal year, as reported in the annual NACUBO Endowment Study (changed to the NACUBO-Common Fund Study of Endowments in 2009). 


\section{Goals Specific to Research Universities}

Academic Quality

Awards include: American Council of Learned Societies (ACLS) Fellows, Beckman Young Investigators, Burroughs Wellcome Fund Career Awards, Cottrell Scholars, Fulbright American Scholars, Getty Scholars in Residence, Guggenheim Fellows, Howard Hughes Medical Institute Investigators, Lasker Medical Research Awards, MacArthur Foundation Fellows, Andrew W. Mellon Foundation Distinguished Achievement Awards, National Endowment for

Faculty Awards the Humanities (NEH) Fellows, National Humanities Center Fellows, National Institutes of Health (NIH) MERIT, National Medal of Science and National Medal of Technology, NSF CAREER awards (excluding those who are also PECASE winners), Newberry Library Longterm Fellows, Pew Scholars in Biomedicine, Presidential Early Career Awards for Scientists and Engineers (PECASE), Robert Wood Johnson Policy Fellows, Searle Scholars, Sloan Research Fellows, Woodrow Wilson Fellows. As reported by the Top American Research Universities - see link.

The number of National Academy members included in the National Academy of Sciences,

\section{National Academy Members}

Number of Post-Doctoral appointees

\section{Number of Science \& Engineering Disciplines nationally ranked in Top 100 for research expenditures}

\section{Return on Investment}

Total Research Expenditures (\$M)

\section{Science \& Engineering Research Expenditures in non-medical/health sciences}

\section{Percent of R\&D Expenditures funded from External Sources}

\section{Patents Issued}

\section{Licenses/Options Executed}

\section{Licensing Income Received (\$M)}

\section{Number of Start-up Companies National rank is higher than predicted by Financial Resources Ranking based on US News \& World Report} Research Doctoral Degrees Awarded
National Academy of Engineering, and the Institute of Medicine. As reported by the Top American Research Universities - see link.

As submitted to the National Science Foundation Survey of Graduate Students and Postdoctorates in Science \& Engineering (also known as the GSS) - see link.

The number of Science \& Engineering disciplines the university ranks in the top 100 (for public and private universities) based on the National Science Foundation's annual survey for R\&D expenditures, which identifies 8 broad disciplines within Science \& Engineering (Computer Science, Engineering, Environmental Science, Life Science, Mathematical Sciences, Physical Sciences, Psychology, and Social Sciences). Historically NSF provided these rankings (see tables 45-61 at link), but now data must be queried via WebCASPAR - see link.

Total expenditures for all research activities (including non-science and engineering activities) as reported in the National Science Foundation annual survey of Higher Education Research and Development (HERD).

This metric reports the Science \& Engineering total R\&D expenditures minus the research expenditures for medical sciences as reported by the National Science Foundation. Historically NSF provided these data (see link, table 36 minus table 52), but now data must be queried via WebCASPAR.

This metric reports the amount of research expenditures that was funded from federal, private industry and other (non-state and non-institutional) sources.

Source: National Science Foundation annual survey of Higher Education Research and Development (HERD).

The number of patents issued in the fiscal year as reported in the 2011-12 Accountability Report (table 6A).

Licenses/options executed in the fiscal year for all technologies as reported in the 2011-12 Accountability Report (table 6A).

License issue fees, payments under options, annual minimums, running royalties, termination payments, amount of equity received when cashed-in, and software and biological material end-user license fees of $\$ 1,000$ or more, but not research funding, patent expense reimbursement, valuation of equity not cashed-in, software and biological material end-user license fees of less than $\$ 1,000$, or trademark licensing royalties from university insignia. Data as reported in the 2012-13 Accountability Report (table 6A).

The number of start-up companies that were dependent upon the licensing of University technology for initiation as reported in the 2012-13 Accountability Report (table 6A).

This metric compares the overall national university ranking to the financial resources rank as reported by the US News and World report.

The number of research doctoral degrees awarded annually as reported in the 2012-13 Accountability Report (table 5B). 


\section{Professional Doctoral Degrees Awarded}

The number of professional doctoral degrees awarded annually as reported in the 2012-13 Accountability Report (table 5B).

\section{Student Debt Summary}

\section{Percent of Bachelor's Recipients with Debt}

\section{Average Amount of Debt for Bachelor's who have graduated with debt}

This is the percentage of bachelor's graduates in a given academic year who entered the university as a first-time-in-college (FTIC) student and who borrowed through any loan programs (institutional, state, Federal Perkins, Federal Stafford Subsidized and unsubsidized, private) that were certified by your institution - excludes parent loans.

Source: Common Dataset (H4).

This is the average amount of cumulative principal borrowed (from any loan program certified by the institution) for each native, FTIC bachelor's recipient in a given academic year that graduated with debt - see metric definition above. This average does NOT include students who did not enter a loan program that was certified by the institution.

Source: Common Dataset (H5).

Student loan cohort default rate (CDR) data includes undergraduate and graduate students, and refers to the three federal fiscal year period when the borrower enters repayment and ends on the second fiscal year following the fiscal year in which the borrower entered repayment. Cohort default rates are based on the number of borrowers who enter repayment, not the number and type of loans that enter repayment. A borrower with multiple loans from the same school whose loans enter repayment during the same cohort fiscal year will be included in the formula only once for that cohort fiscal year. Default rate debt includes: Federal Stafford Loans, and Direct Stafford/Ford Loans - for more information see:

http://ifap.ed.gov/DefaultManagement/CDRGuideMaster.html.

\begin{tabular}{|c|c|c|c|}
\hline \multicolumn{4}{|c|}{ Three Year CDR } \\
\hline $\begin{array}{l}\text { Cohort } \\
\text { Fiscal } \\
\text { Year }\end{array}$ & $\begin{array}{c}\text { Year } \\
\text { Published }\end{array}$ & $\begin{array}{l}\text { Borrowers in the Numerator } \\
\text { Borrowers in the Denominator }\end{array}$ & $\begin{array}{l}\frac{\text { 3-Yr Time Period }}{\text { (Numerator) }} \\
\text { 1-Yr Time Period } \\
\text { (Denominator) }\end{array}$ \\
\hline 2009 & 2012 & $\begin{array}{c}\text { Borrowers who entered repayment in } 2009 \\
\text { and defaulted in 2009, } 2010 \text { or } 2011 \\
\text { Borrowers who entered repayment in } 2009\end{array}$ & $\frac{10 / 01 / 2008 \text { to } 9 / 30 / 2011}{10 / 01 / 2008 \text { to } 9 / 30 / 2009}$ \\
\hline 2010 & 2013 & $\begin{array}{l}\text { Borrowers who entered repayment in } 2010 \\
\text { and defaulted in } 2010,2011 \text { or } 2012 \\
\text { Borrowers who entered repayment in } 2010\end{array}$ & $\frac{10 / 01 / 2009 \text { to } 9 / 30 / 2012}{10 / 01 / 2009 \text { to } 9 / 30 / 2010}$ \\
\hline 2011 & $2014 *$ & $\begin{array}{l}\text { Borrowers who entered repayment in } 2011 \\
\text { and defaulted in } 2011,2012 \text { or } 2013 \\
\text { Borrowers who entered repayment in } 2011\end{array}$ & $\frac{10 / 01 / 2010 \text { to } 9 / 30 / 2013}{10 / 01 / 2010 \text { to } 9 / 30 / 2011}$ \\
\hline 2012 & 2015 & $\begin{array}{l}\text { Borrowers who entered repayment in } 2012 \\
\text { and defaulted in 2012, } 2013 \text { or } 2014 \\
\text { Borrowers who entered repayment in } 2012\end{array}$ & $\frac{10 / 01 / 2011 \text { to } 9 / 30 / 2014}{10 / 01 / 2011 \text { to } 9 / 30 / 2012}$ \\
\hline 2013 & 2016 & $\begin{array}{l}\text { Borrowers who entered repayment in } 2013 \\
\text { and defaulted in } 2013,2014 \text { or } 2015 \\
\text { Borrowers who entered repayment in } 2013\end{array}$ & $\frac{10 / 01 / 2012 \text { to } 9 / 30 / 2015}{10 / 01 / 2012 \text { to } 9 / 30 / 2013}$ \\
\hline 2014 & 2017 & $\begin{array}{l}\text { Borrowers who entered repayment in } 2014 \\
\text { and defaulted in } 2014,2015 \text { or } 2016 \\
\text { Borrowers who entered repayment in } 2014\end{array}$ & $\frac{10 / 01 / 2013 \text { to } 9 / 30 / 2016}{10 / 01 / 2013 \text { to } 9 / 30 / 2014}$ \\
\hline 2015 & 2018 & $\begin{array}{l}\text { Borrowers who entered repayment in } 2015 \\
\text { and defaulted in } 2015,2016 \text { or } 2017 \\
\text { Borrowers who entered repayment in } 2015\end{array}$ & $\frac{10 / 01 / 2014 \text { to } 9 / 30 / 2017}{10 / 01 / 2014 \text { to } 9 / 30 / 2015}$ \\
\hline
\end{tabular}

